\title{
DUET - The Dark Universe Exploration Telescope
}

\author{
K. JAHODA FOR THE DUET COLLABORATION \\ Laboratory for High Energy Astrophysics, Goddard Space Flight Center, Greenbelt MD 20771, USA
}

Received date will be inserted by the editor; accepted date will be inserted by the editor

\begin{abstract}
DUET, a proposed NASA Midex class mission, will produce a catalog of $\sim 20,000$ X-ray selected clusters and groups of galaxies suitable for detailed cosmological investigations. We present the cosmological constraints achievable by DUET, the functional requirements of the survey, and briefly describe an instrument/mission design capable of achieving these requirements. The advantages of an X-ray survey for constraining the nature and amount of dark energy and dark matter are emphasized. Constraints on cosmological parameters are precise and complementary to other approaches. The density of dark energy, dark matter, and neutrinos will be measured with a statistical uncertainty of $0.5 \%, 0.5 \%$, and $0.2 \%$ of the critical density and the dark energy equation of state can be measured to \pm 0.2 if dark energy and dark matter contribute $\sim 2 / 3$ and $\sim 1 / 3$ of the critical density as currently believed. DUET is a multi-national collaboration led by GSFC with substantial contributions from groups in the US, Europe, and Japan.
\end{abstract}

\section{Introduction}

The Dark Universe Exploration Telescope (DUET) is a MIDEX class concept that would study the distribution and nature of dark energy and dark matter using two large and deep X-ray selected samples of clusters of galaxies. A "wide" sample would reach limiting $0.5-2 \mathrm{keV}$ fluxes of $5 \times$ $10^{-14} \mathrm{erg} \mathrm{s}^{-1} \mathrm{~cm}^{-2}$ over the $10^{4} \mathrm{deg}^{2}$ region of the Sloan Digital Sky Survey (SDSS). A "deep" sample would reach a limiting flux 10 times fainter over $100 \mathrm{deg}^{2}$ in a southern hemisphere region that overlaps a deep Sunyaev-Zeldovich (SZ) survey. These catalogs can be obtained within a three year mission. The wide sample will contain 15-20,000 clusters or groups of galaxies; the deeper survey will provide significant constraints on cluster evolution. Together the two samples will provide powerful probes of cosmological models, precise estimates of fundamental cosmological parameters, and clues to the nature of Dark Energy and Dark Matter. The expected constraints are highly complementary and provide similar or better statistical precision as those expected from the next generation of Cosmic Microwave Background experiments and the proposed SuperNova Acceleration Probe (SNAP).

DUET was proposed to the NASA MIDEX competition in 2001 by an international collaboration led by the Goddard Space Flight Center (GSFC) with flight hadware, ground system, and optical follow-up contributions from GSFC, the Massachusetts Institute of Technology, the European Space Agency, the Italian (ASI) and Japanese (ISAS) Space Agen-

Correspondence to: keith.jahoda@gsfc.nasa.gov cies, and the Universities of Illinois, Chicago, and Hawaii. The Science Team includes members from these institutions and Berkeley, Cal Tech, Princeton, ESTEC, Leicester, MPE, Brera, Saclay, Santander, TiTech, and Trieste.

\section{From Clusters to Cosmology}

Current cosmological theories make precise predictions about the "halo mass function" as a function of cosmological parameters (i.e. the Hubble constant $H_{o}$, the matter density $\Omega_{M}$, the dark energy density $\Omega_{E}$, the fluctuation amplitude $\sigma_{8}$ ) and redshift. The space density and distribution of the most massive objects, which are observationally identified with clusters of galaxies, are very sensitive to cosmological parameters. Thus, a large catalog of clusters with a well defined and uniform selection function and a robust observable-mass relationship provides an excellent probe of cosmological parameters Haiman, Mohr, and Holder 2001. Levine, Schulz, and White 2002).

The basic observational data provided by DUET is a cata$\log$ of about 20,000 clusters described by spatial coordinates, angular size, and X-ray flux; for the brightest $\sim 1000$ objects, DUET will also measure temperatures. Correlation with optical observations, either the SDSS or dedicated follow-up, provides redshift information allowing a reliable determination of X-ray luminosity. The mass of each cluster can be estimated from the $L-M$ relationship (fig. 1) which is well defined over a broad range of cluster masses and luminosities Reiprich and Boehringer 2002. The observed distribution of masses can be compared with cosmological predic- 


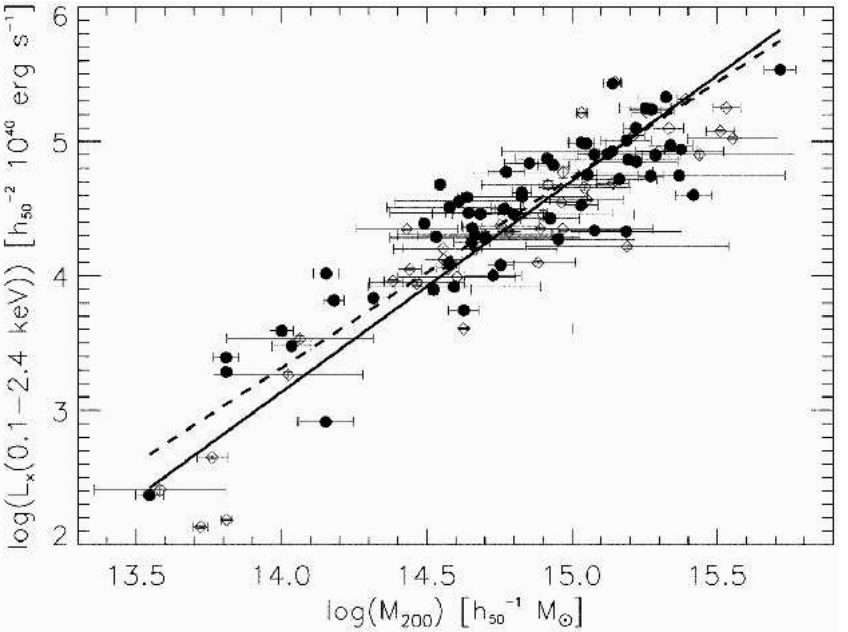

Fig. 1. The Luminosity-Mass relationship for a large sample of clusters of galaxies observed by ROSAT and ASCA from Reiprich and Boehringer 2002. The scatter is $60 \%$ in mass at a fixed luminosity, which dominates statistical errors in estimating the mass.

tions; there are numerous checks on the mass scale including the DUET temeperatures for the brightest objects, comparison with detailed Chandra and XMM-Newton observations, correlations with masses derived from SZ, strong and weak lensing, and velocity dispersion surveys.

$\mathrm{X}$-rays are an excellent cluster tracer since most of the baryonic (i.e. potentially visible matter) resides in a hot diffuse and X-ray emitting gas which permeates the potential well of the cluster. The temperature of the gas is primarily determined by gravitational heating. Although the $L-M$ relationship is steeper than predicted from gravitational heating alone, it is well defined and predictive over several orders of magnitude in both quantities. Several pre-heating models are capable of explaining the observed $L-M$ relationship, and the complete physics based description is likely to be well understood in detail prior to the DUET mission.

Clusters are bright objects in the X-ray sky, and at high galactic latitudes are the only common extended sources. They are easy to identify from X-ray data with positions adequate to provide optical identifications and redshifts.

\section{Constraints}

Precise cosmological inferences can be made by statistical analyses of the DUET three dimensional map of the distribution of clusters of galaxies. Figures 3 through 5 show examples. These figures are based on simulations using a fiducial cosmology 17 and demonstrate the precision with which parameters can be determined. The error contours are small, and

\footnotetext{
1 The fiducial cosmology for the simulations presented here assumes a total matter density $\Omega_{M}=0.3$, a dark energy density $\Omega_{E}=$ 0.7 , a Hubble constant of $100 h \mathrm{~km} \mathrm{~s}^{-1} \mathrm{Mpc}^{-1}$ with $h=0.72$, a baryon density $\Omega_{b} h^{2}=0.02$, a dark energy equation of state parameter $w=-1$, a power spectrum normalization $\sigma_{8}=1.0$, a fluctuation spectrum index $n_{s}=1$, and a neutrino density $\Omega_{\nu}=0$.
}

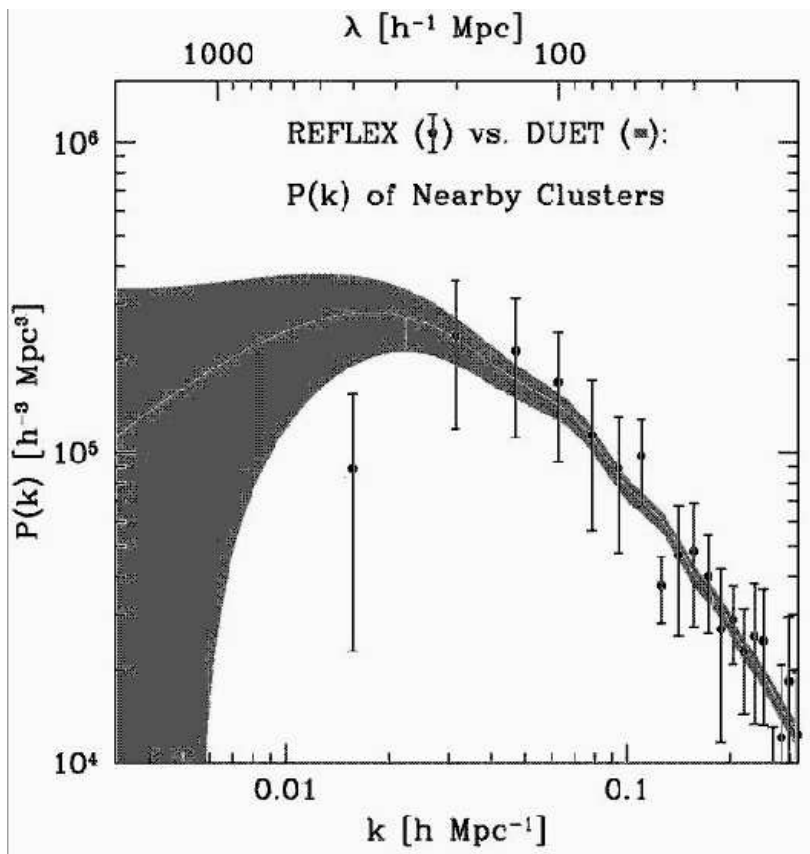

Fig. 2. Comparison of the REFLEX power spectrum with the DUET power spectrum for clusters with redshift less than 0.25 . DUET will measure independent power spectra, of similar statististical precision, for the redshift range 0.25 to 0.45 and for redshifts above 0.45 .

they are significantly tilted with respect to the contours that will be determined by other planned or proposed experiments such as Planck and SNAP (fig. 5). This means that the combined experiments will either determine with great precision the values of many cosmological parameters contained in currently popular cosmological models, or more excitingly, reveal discrepancies with CMB/SNAP, and thus point to a need for a fundamental reassesment of cosmological models

Two powerful statistical tests are the power spectrum, $P(k)$, and the redshift distribution, $d N / d z$. The state of the art in measurement of the power spectrum is derived from the REFLEX catalog (Boehinger et al. 2001) with almost 500 objects in the southern sky. DUET will provide 40 times more objects in a comparable solid angle. Figure 2 pares the power spectrum measured from the REFLEX survey with that expected from DUET clusters with redshift below 0.25 (i.e. about one third of the DUET survey selected in the redshift range sampled by REFLEX.) Interpretation of both $d N / d z$ and $P(k)$ benefits enormously from a uniform and well defined survey. Although XMM-Newton will discover a substantial number of clusters serendipitously (Romer et al. 2001), and provide information complimentary to DUET, the XMM serendipitous catalog will be constructed from numerous pencil beam observations of various depths, will generally probe fainter fluxes (and lower masses), will often observe directions not covered by the SDSS, and does not have a dedicated optical follow-up program to provide redshifts for the balance of the clusters. 


\subsection{Power Spectrum}

The power spectrum measures, as a function of scale, the lumpiness of the universe (Borgani and Guzzo 2001) and provides a more complete description than $\sigma_{8}$ ( the variance of the density field on $8 \mathrm{Mpc}$ scales) which is often used to normalize the power spectrum and which can be related to $\mathrm{P}\left(0.2 h \mathrm{Mpc}^{-1}\right)$ (Henry 2002).

Even in the context of cosmological models dominated by dark matter and dark energy, the overall shape of the power spectrum is quite sensitive to small amounts of baryons or neutrinos, whose clustering properties are different than for cold dark matter alone. For baryons, the effects arise from numerous non-gravitational interactions including acoustic oscillations (which produce the beautiful peaks in the microwave background power spectra) and Compton drag (see Eisenstein and $\mathrm{Hu} 1998$ for a detailed description). A neutrino (or Hot Dark Matter) contribution to the energy density of the universe would suppress structure at scales below $k>\approx 0.05 h \mathrm{Mpc}^{-1}$ (Elgaroy et al. 2002). Figure B shows that DUET could provide an interesting upper limit to the energy density in neutrinos (and thus neutrino mass). The lower limit in the figure comes from Super-Kamiokande results Ahmad et al. 2001.

\subsection{Redshift Distribution}

For a non-evolving tracer, the redshift distribution $d N / d z$ is a probe of cosmological volume (and thus of the energy density of dark energy and dark matter). The ideal tracer has a constant (or calculable) volume density and a well known selection function. X-ray selected clusters of galaxies satisfy these conditions. While clusters of galaxies do evolve, their growth is dominated by gravitational collapse. This makes the volume density directly calculable without including the difficult physics involved in galaxy formation. Recent numerical simulations demonstrate the existence of a universal mass function, describable in terms of cosmological parameters and the cluster growth function for a wide range of cosmological models (Jenkins et al. 2001). Evolution of the cluster volume density provides tight constraints as discussed in detail detail by Haiman, Mohr, and Holder 2001 and demonstrated in figures 4 and 5 .

\section{DUET Pathfinder}

The ROSAT North Ecliptic Pole (NEP) survey Henry et al. 2001 has measured the X-ray flux distribution of clusters, and demonstrates in a model independent way that DUET will detect $15-20,000$ clusters in the SDSS region. The NEP survey covered $81 \mathrm{deg}^{2}$ at the north ecliptic pole and reached a limiting sensitivity of a few $10^{-14} \mathrm{erg} \mathrm{s}^{-1} \mathrm{~cm}^{-2}$. An eight year optical follow-up effort identified all but 2 of the 445 sources. The survey includes 64 clusters with median and maximum redshift of 0.205 and 0.811 .

The complete identification of NEP sources allows quantitative statements about the optical magnitudes of the

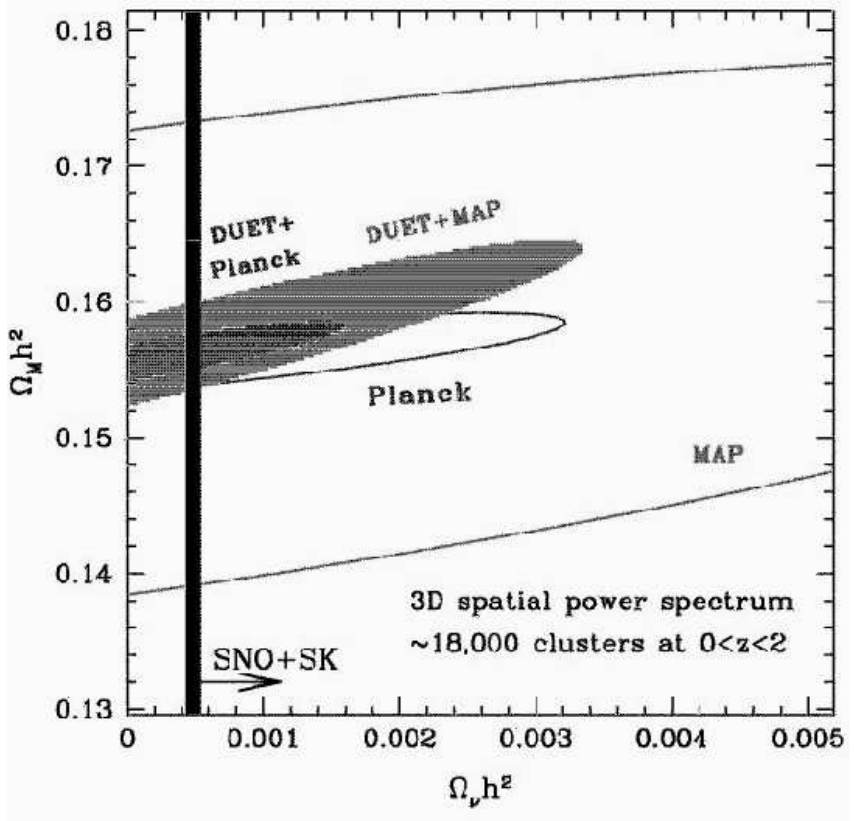

Fig. 3. DUET constraints on the energy density of neutrinos. The shape of the power spectrum depends on the fraction of clustered and non-clustered matter. Neutrinos are not expected to cluster on the scales of clusters of galaxies; DUET can measure the presence of non clustered dark matter at a few tenths of a percent of the critical density.

sources. Mullis (2001) finds that the median plus $1 \sigma \mathrm{B}$ magnitude for the AGN is 20.7 (i.e. 190 of the 218 AGN are brighter than this), and all of the AGN have B magnitudes less than 22. Stars also have magnitudes as faint as 22 , but the mean plus $1 \sigma$ point ( 137 of 152 ) is at $\mathrm{B}=17.2$. The details of the DUET stellar content are expected to be latitude dependent. The depth of the SDSS photometric survey is well suited to identify the vast majority of the X-ray point sources in the DUET catalog, in addition to providing redshifts for the clusters.

\section{Requirements of an X-ray Survey}

The observational requirements of DUET collectively dictate the size and quality of the X-ray optics and focal plane. Required features include

- The ability to distinguish extent from X-ray data alone, in order to separate clusters of galaxies from other the high latitude sources which are pointlike

- Small enough error circles to provide unambiguous identifications

- Small systematic mass errors

- Sufficient grasp to obtain a large catalog within the scope of a MIDEX mission

Requiring 50 photons for threshold objects, in combination with the proposed optics, satisfies the first two requirements. The tight observed $L-M$ relationship and the ability to calibrate DUET masses against detailed Chandra and XMM-Newton observations, and against masses derived 


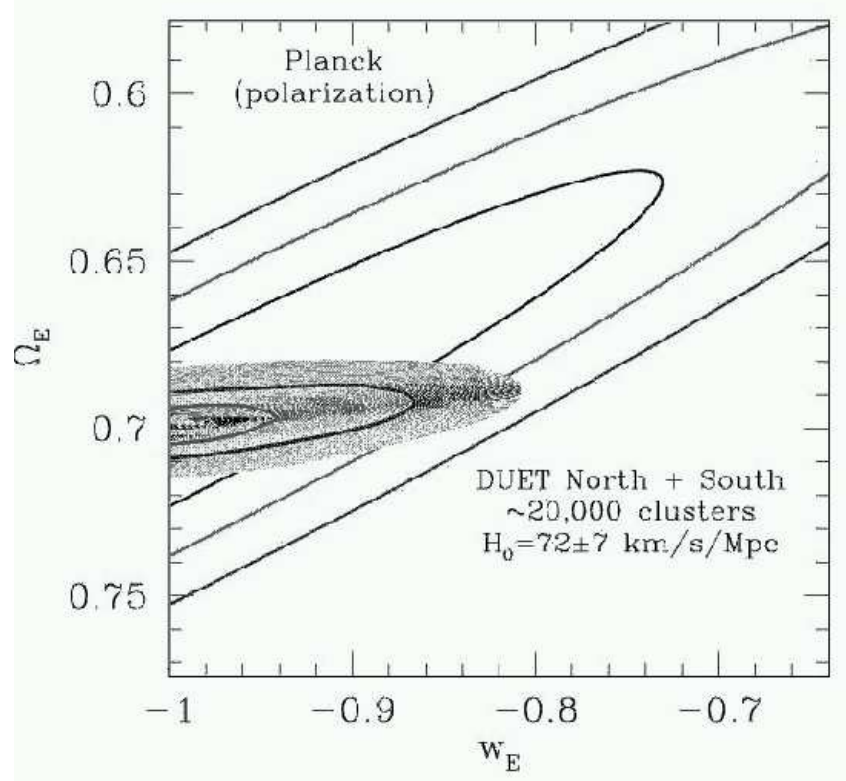

Fig. 4. DUET constraints on the Dark energy density and the dark energy equation of state parameter $w$ assuming a flat universe; the contours are marginalized over $\sigma_{8}$ and $H_{o}$.

from SZ, strong and weak lensing, and velocity distpersions satisfies the third. The grasp available from the flight spare mirror for the X-ray Multi-Mirror (XMM) mission can achieve these results in a three year mission.

The XMM mirror has substantial effective area out to a radius of 30', albeit with degraded angular resolution. Weighted sums of ray tracing results allow the investigation of the field of view averaged point spread function, which has a $90 \%$ encircled energy radius of 46 arcsec, and a vignetting relative to the on-axis effective area of 0.3 . We have performed simulations under the assumption that 50 photons are detected from a source with a $\beta$ model surface brightness profile (Cavaliere and Fusco-Femiano 1976); we assume $\beta=0.7$. The radial profile of the source photons (including appropriately scaled contributions from instrumental and cosmic backgrounds) are compared to the point spread function, and a Kolmogorov-Smirnov test is employed to determine whether the source is extended. 50 photons are sufficient to identify ( $99 \%$ confidence level) 70,92 , and $98 \%$ of clusters with core radii of 20,30 , and 40 arcsec respectively.

The typical core radius for clusters is $220 \mathrm{kpc}$ Vikhlinin et al. 1999) and ROSAT samples suggest that core radii do not evolve (Vikhlinin et al. 1998), suggesting that the typical cluster has a minimum angular core-radius of 30 arcsec for redshifts near 1 in flat universes. Even if core-radii evolve, the XMM mirror should detect as extended the large majority of clusters, with a calculable and calibratable incompleteness for the smaller systems.

\section{DUET Instrument Implementation}

The DUET concept takes advantage of the XMM flight spare mirror, i.e. an excellent optic which already exists and which

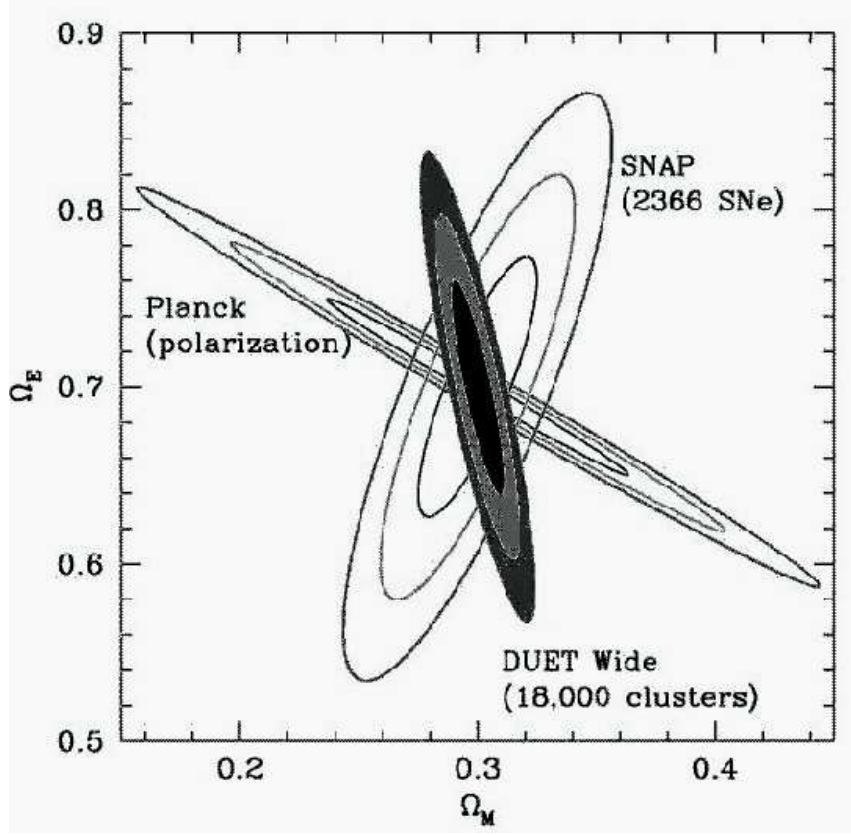

Fig. 5. DUET constraints on the matter and dark energy density (marginalized over $w$ and $H_{o}$ ), compared with measurements from the CMB and the proposed SNAP survey. Note that the DUET constraints have similar precision while being highly complementary to the other constraints.

is well calibrated. The desired field of view is 1 degree (diameter). The focal length is $7.5 \mathrm{~m}$. The field of view is achieved with a 4 by 4 array of CCD chips while the focal length is accommodated by a folding optical bench.

The optical bench consists of two triangular truss sections constructed from flat composite panels connected by a hinge and latch system designed to be operated once, after a suitable orbit is achieved. The instrument can be accommodated on a modified Ball Aerospace BCP 2000 spacecraft bus and a Delta II launch vehicle.

The SDSS will provide redshifts for $85 \%$ of the clusters. A dedicated 3 year optical followup program will complete the DUET catalog.

Acknowledgements. I thank the organizers of the X-ray Surveys conference for a stimulating conference. I also thank my collaborators on the DUET proposal who have contributed to the concepts and simulations presented in this summary.

\section{References}

Ahmed, Q.R. et al. 2001, Phys. Rev. Lett., 87, 071301

Boehringer et al.: 2001, A\&A, 369, 826

Borgani, S. and Guzzo, L.: 2001, Nature, 409, 39

Cavaliere, A. and Fusco-Femiano, R.: 1976, A\&A, 49, 137

Eisenstein, D.J. and Hu, W.: 1998, ApJ, 496, 605

Elgaroy, O. et al.: 2002, Phys. Rev. Lett., 89, 061301

Haiman, Z., Mohr, J.J., and Holder, G.P.: 2001, ApJ, 553, 545

Henry, J. P.: astro-ph/0207148, in press in Matter and Energy in Clusters of Galaxies, ASP Conference Series, Hwang and Bowyer, eds.

Henry, J.P. et al.: 2001, ApJL, 553, L109

Jenkins, A.: 2001, MNRAS, 321, 372 
Levine, E.S., Schulz, A.E. and White,M.: 2002, astro-ph/0204273

Mullis, C.R.: 2001, Ph.D. thesis, U. HI

Reiprich, T., Boehringer, H.: 2002, ApJ, 567, 716

Romer, A.K. et al.: 2001, ApJ, 547, 594

Vikhlinin, A. et al.: 1998, ApJL, 498, L21

Vikhlinin, A. et al.: 1999, ApJ, 525, 47 